\title{
DVB-T2 simulation model for OPNET Poster abstract
}

\author{
Adlen Ksentini \\ IRISA - University of Rennes 1 \\ Campus universitaire de Beaulieu \\ Rennes, France \\ adlen.ksentini@irisa.fr
}

\author{
Yassine Hadjadj-Aoul \\ IRISA - University of Rennes 1 \\ Campus universitaire de Beaulieu \\ Rennes, France \\ yassine.hadjadj-aoul@irisa.fr
}

\begin{abstract}
DVB-T2 is offering a new way for broadcasting value-added services to end users, such as High Definition (HD) TV and 3D TV. Thanks to the advances made in digital signal processing, and specifically in channel coding, DVB-T2 brings an increased transfer capacity of $50 \%$ and a new flexibility in services' broadcasting in contrast with the first generation DVB-T standard. As DVB-T2 is still in deployment's test, simulation model could be an interesting way to evaluate the performance of this network in supporting new valueadded services. In this paper, we describe the new features and enhancements we have integrated within the DVB-T2 module in OPNET, and in particular: (i) realistic physical model;(ii) MPEG-TS layer with an IP encapsulator;(iii) hierarchical application layer ables to use pcap traces to simulate real video traces. Finally, we validate the developed DVB-T2 model according to the standard recommendation in terms of physical layer performance (BER versus SNR).
\end{abstract}

\section{Keywords}

DVB-T2, Broadcast, simulation, OPNET

\section{INTRODUCTION}

DVB-T2 [1] is the second generation standard for digital terrestrial television broadcasting. Thanks to the advances made in digital signal processing, and specifically in channel coding, DVB-T2 brings an increased transfer capacity of $50 \%$ and a new flexibility in services' broadcasting when compared to the first generation DVB-T standard published in 1997. To allow a high robustness against multipath propagation, DVB-T2 uses a Coded Orthogonal Frequency Division Multiplexing (COFDM) multi-carrier modulation, in a similar way to DVB-T. A wider range of schemes is proposed, from $1 \mathrm{~K}$ carrier up to $32 \mathrm{~K}$ carrier, to meet the requirements of a wide range of receiving situations (fixed and mobile) and network topologies (single and multiple frequency networks). In terms of channel coding, DVB-T2 uses Low Density Parity Check (LDPC) block codes and

\footnotetext{
Permission to make digital or hard copies of all or part of this work for personal or classroom use is granted without fee provided that copies are not made or distributed for profit or commercial advantage and that copies bear this notice and the full citation on the first page. To copy otherwise, to republish, to post on servers or to redistribute to lists, requires prior specific permission and/or a fee.

Simutools 2012, March 19-23, Desenzano del Garda, Italy

Copyright @ 2012 ICST 978-1-936968-47-3

DOI 10.4108/icst.simutools.2012.247750
}

Bose-Chaudhui-Hochquenghene ( $\mathrm{BCH}$ ) coding, which provide more robust error correction than the convolutional and Reed Solomon encoding used in DVB-T. Most of the capacity gain of DVB-T2 comes from this fundamental change of channel coding. The DVB-T2 physical layer data channel is divided into logical entities called the Physical Layer Pipes (PLP). Each PLP carries one logical data stream. Examples of such a logical data stream, would be an audio-visual multimedia stream along with the associated signaling information, or an hierarchical application streams, which can address at the same time different qualities as it is the case for Scalable Video Coding (SVC) [2]. The PLP architecture is designated to be flexible so that arbitrary adjustments of robustness and capacity can be easily done. Thus, using different PLP enables broadcasting of multiple services or groups of services with separate channel coding and modulation settings on a single radio channel. Broadcasting several service components over the same channel is thus made possible, with differentiated levels of robustness, which was not possible with the previous DVB-T standard. Regarding the upper layers, the DVB-T2 provides two main IP encapsulation protocols, the MPEG-TS [3] packetization, which has been the classical encapsulation scheme for DVB services, and the Generic Stream Encapsulation (GSE) [4], which was designated to provide appropriate encapsulation for IP traffic.

Simulation tools, meanwhile, are a good mean to evaluate the performance of a system in different conditions. This is also useful to dimension a system and optimize its performance, which represent an interesting issues, particularly for DVB-T2 network as it is still in test. Among the existing network simulation tools, OPNET [5] is one of the leading simulator that provides powerful simulation capability for testing networks architectures and protocols. It includes simulation models from the application layer to the lower layers of networks, including wired and wireless connection. Reader can refer to [6], for an interesting comparison of several computer simulations, with a brief focus on OPNET. In this work, we describe the features and enhancements we added to support DVB-T2 simulation in OPNET. The most important features we introduced are: (1) realistic physical layer that uses measured Bit Error Rate (BER) versus Signal-to-Noise Ratio (SNR) traces for different modulation schemes; (2) MPEG-TS layer, with an IP encapsulator based on Unidirectional Lightweight Encapsulation (ULE) [7]; (3) application layer that supports hierarchical streams, and associates each stream with a PLP. Furthermore, the developed application layer is able to parse pcap traces to simu- 


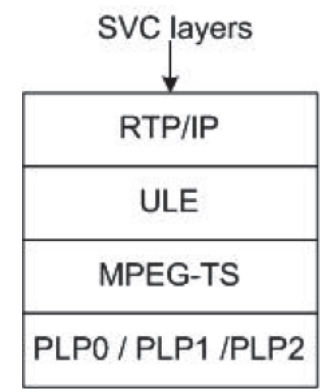

Figure 1: Layered DVB-T2 model OPNET

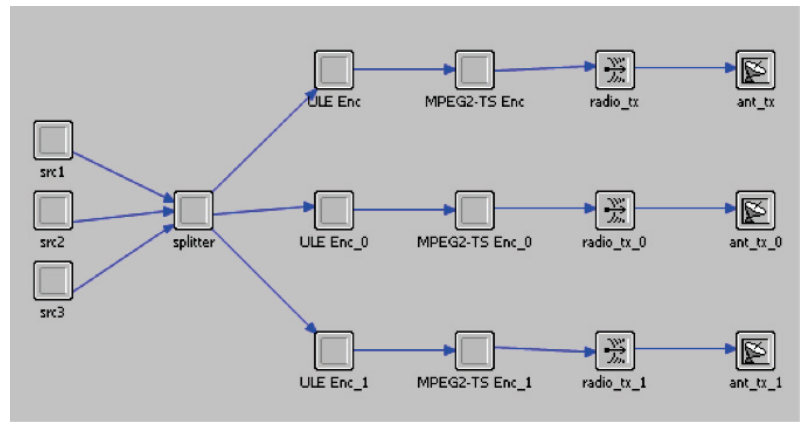

Figure 2: Transmitter node model

late real video traffics. The purpose of this simulation model is to enable the evaluation, in a realistic environment, the capacity of DVB-T2 to support new services, such as High Definition video broadcast, for both fixed and mobile receivers. To the best of our knowledge this is the first simulator platform for DVB-T2 networks. Note that, this DVB-T2 simulation model could be extended easily to simulate DVB-H or DVB-S2, which share many features with DVB-T2 (only the physical layer that needs modification).

This paper is organized as follow: section 2 presents the developed DVB-T2 model in OPNET. In section 3, we validate the physical layer model regarding the Nordig recommendations [9]. We conclude this paper in section 4.

\section{DVB-T2 OPNET MODEL}

\subsection{Model Overview}

To simulate and evaluate the performance of DVB-T2 network, we have introduced new features and enhanced other available features in OPNET. The proposed model is able to simulate the entire DVB-T2 chain, by including models for the DVB-T2 broadcast gateway (transmitter) as well as fixed and mobile receivers. Each entity includes the IP/ULE/MPEGTS/PLP transmit/receiver capability as showed in Figure 1. Herein, we describe the main features we introduced to support DVB-T2 in OPNET. For more details on the entire model, reader can refer to [10].

\section{TRANSMITTER MODEL (DVB-T2 GATE- WAY)}

Figure 2 presents the design architecture of the transmitter node model. This model consists of the application source, the data splitter, the MPEG-TS layer (including the

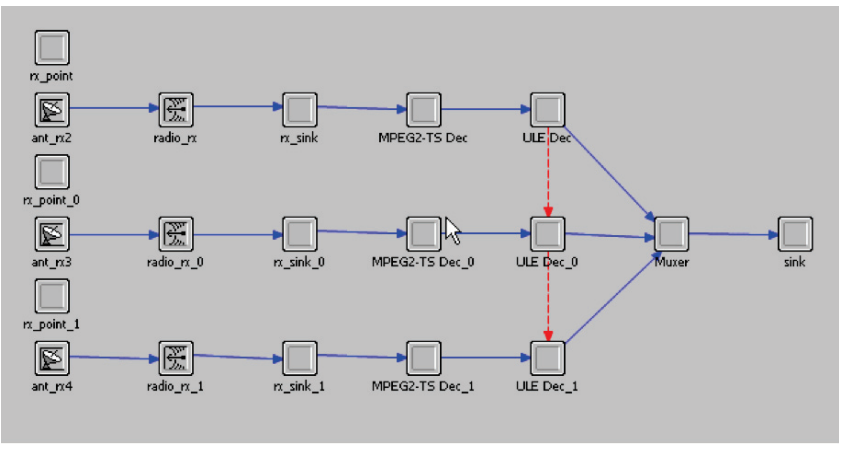

Figure 3: Receiver node model

IP encapsulator) and the physical layer. For this model, the physical layer is using three physical layer pipelines (PLP) (the number of PLP is not fixed, it can be extended to $n$ PLP); each PLP has its own physical modulation and FEC protection. To configure one PLP, we have to extend its attributes. Thus, it is possible to define the available physical modulations (16QAM1/2, 64QAM3/5, 256QAM3/4, $256 Q A M 3 / 5$ and $256 Q A M 5 / 6)$, the frequency, data rate and the power of transmission for each PLP. Concerning the application, there is three sources attached to the splitter, which represents, for instance, three applications sources (audio/video), or one scalable application like SVC. The main functionality of the splitter is to redirect each application layer data to its associated PLP. Then these data are fragmented (if they exceed the 1500Bytes) in IP packets. To transmit an IP packet in the DVB chain, ULE encapsulation is used. Therefore, each IP packet is encapsulated in ULE packet. Here, it is important to note that the standard way to carry IP datagrams over MPEG2-TS are multiprotocole Encapsulation (MPE) [8] and ULE.

An alternative to MPEG2 TS, for encapsulating IP packets, is Generic Stream Encapsulation (GSE). GSE encapsulation reduces the overhead by a factor of 2 to 3 times when compared to MPEG-TS. However, GSE is not well deployed in the DVB-T2 products. For this simulation model we decided to implement the ULE encapsulation mechanism, since it reduce overhead and ensure more functionalities than MPE. Each ULE packet is then sent to the MPEGTS layer. This layer will fragment each ULE Packet into different MPEG-TS packets. The size of MPEG-TS packet is 188Bytes. After that, these packets are directed to the PLP, which transmit them over the radio channel. Note that, each PLP is associated with an antenna. The same antenna model is used by the three PLPs. The antenna model used for transmission is an Omni directional antenna.

\section{RECEIVER MODEL}

Figure 3 shows the architecture design of the receiver node model.This model is used either to simulate a fix or mobile station. The receiver node model is asymmetric to the transmitter node model, it is constituted by the same layers (application, MPEG-TS and physical layer). The radio receiver (PLP) begins by receiving the broadcasted frame. Then the received frame is sent to the MPEG-TS layer, which waits for a number of packets to re-constitute an ULE packet. The ULE layer receives the ULE packet and extracts the IP packet, which is directed to the muxer. This entity (muxer) 


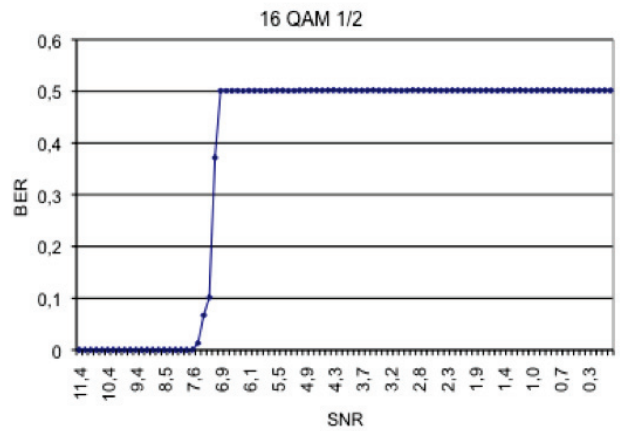

Figure 4: 16 QAM 1/2 Simulated BER vs SNR

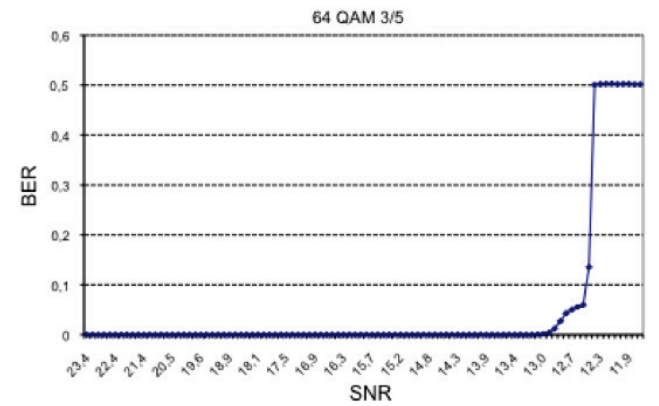

Figure 5: 64 QAM 3/5 Simulated BER vs SNR

will receive the three applications streams sent through the three PLP, and reconstitute the application stream. The main differences between the transmitter and the receiver models are:

- The antenna model, where a directional antenna is used in case of fix receiver, and an omnidirectional antenna for mobile receivers;

- The muxer, which reconstitutes the application streams from different PLPs.

\section{PHYSICAL MODEL VALIDATION}

In order to validate the simulation model, we compared the performance of the physical channel results against the nordig recommendations. Indeed, these recommendations specify the SNR value from which the BER must appear for each physical modulations. Each commercial DVB-T2 receiver must satisfy these requirements. In this way, we show in Figures 4-5-6-7 the BER versus SNR for 5 physical modulations, namely $16 Q A M 1 / 2,64 Q A M 3 / 5,254 Q A M 3 / 4$ and $256 Q A M 3 / 5$. From these figures, it appears clearly that the physical layer model respects the recommendation of NorDig [9] for DVB-T2 receivers. For instance, the NorDig recommends that in case of Gaussian channel and 16QAM1/2 modulation, the BER is about $10^{-7}$ when the SNR reaches $6 d b$, which is practically respected by our simulation model (see Figure 9). The same behavior is noticed for the other modulations, where our physical model respects the recommendation of the NorDig.

\section{REFERENCES}

[1] "DVB-T2 - the HDTV generation of terrestrial DTV", DVB-TM-3997, MArch 2008.

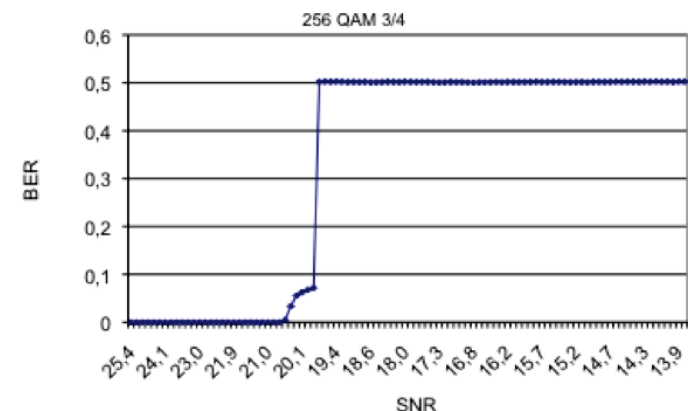

Figure 6: 256 QAM 3/4 Simulated BER vs SNR

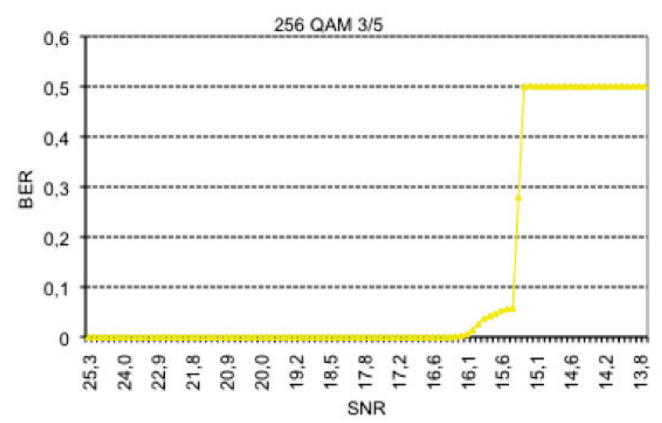

Figure 7: 256 QAM 3/5 Simulated BER vs SNR

[2] Scalable Video Coding, Joint ITU-T Rec. H.264| ISO/IEC 14496-10 / Amd.3 Scalable Video Coding, November 2007.

[3] ISO/IECC IS 13818-1,"Information technology - generic coding of moving pictures and associated audio information: systems".

[4] ETSI TS 102606 V.1.1.1, "Digital Video Broadcasting (DVB); Generic Stream Encapsulation".

[5] www.opnet.com/products/opnet-products.html.

[6] X. J. Chang,"Network simulations with OPNET", Simulation Conference Proceedings 99, pp. 307-314, December 1999.

[7] IETF RFC 4326, G. Fairhurst, and B. Collini-Nocker, "Unidirectional Lighweight Encapsulation (ULE) for Transmission of IP Datagrams over MPEG-2 Transport Stream (TS)", December 2005.

[8] ETSI EN 301192 V 1.4.2, "Digital Video Broadcasting (DVB); DVB specification for data broadcasting", April 2008.

[9] Requierement to Nordog T2 compliant IRDs, Addendum to the Nordig Requierements.

[10] A. Ksentini, Y. Hadjadj-Aoul, "DVB-T2 simulation model in opnet", IRISA internal report n1967, Feb. 2011, Available at http://hal.inria.fr/inria-00567546/PDF/PI-1967.pdf. 